\title{
Performance Analysis of Routing Protocols in Manets
}

\author{
Ahmed M. Jasim \\ Assistant Lecturer, Department of computer engineering, College of Engineering, Diyala University \\ ahmed.1985m@yahoo.com
}

\begin{abstract}
Mobile ad hoc networks (MANETs) are characterized by the absence of centralized management and the lack of any infrastructures. The frequent topology changes and variable propagation conditions make a routing is the most important issue, therefore, it becomes necessary for nodes within the networks to use appropriate routing algorithms. In this paper, we evaluate the performance of AODV, DSR, OLSR and GRP routing protocols using OPNET modeler 14.5 simulation software. In the simulated mobile ad hoc network, we made all nodes receiving FTP traffic from a FTP server as common source. we increased the possibility of multihop routes between nodes with each other or from the server to the nodes by placing them randomly in the network. Our simulations have suggested many of the scenarios to all selected protocols in the identical environmental conditions so as to evaluate their performance with respect to routing overhead, throughput, end-to-end delay and routing load.
\end{abstract}

Keywords: MANET, OLSR, AODV, DSR, GRP.

Paper History: Received: (15/1/2017), Accepted: (14/3/2017)

\section{I- INTRODUCTION}

The usage of wireless computing networks has increased dramatically through radio channels as the transmission medium. In many fields of national economy is seen an increase in demand for mobile portative computers, which would allow to share information with each other and gain access to the global Internet network without a wired connection. Today one of the most rapidly developing directions in wireless computer networks is to create an Ad hoc network. It is a wireless networking without infrastructure. MANET is a kind of ad hoc networks. MANET is a group of mobile nodes interconnecting with each other on peer to peer. An increased interest in MANET is caused by such properties of MANET networks as the simplicity of organization and the free movement of wireless nodes. MANET network can be created in minutes, and include dozens or even hundreds of wireless nodes. A
MANET include mobile nodes and also can include both fixed and mobile nodes. Nodes can be acted as both routers and hosts and connect with each other randomly, forming arbitrary topologies [1]. Such fast change topology makes many of research challenges, the most important one is the routing. Thus, the wireless networks without fixed infrastructure of ad hoc class are radically different from wired and cellular networks. Considered features make the task of providing efficient data transfer extremely difficult, owing to what is necessary to seek effective solutions to problems of network management and information transfer.

The various advantages of MANET, include ease and speed of deployment, independent of previously established infrastructure, selforganizing and adaptive, formed immediately without installing base stations and system administrators' participation, that make them use in all places where infrastructure doesn't exist or damaged and where fast deployment of a computer network is required, in disaster areas, reach areas, during military operations, emergency searches, rescue operations, unscheduled meetings, conferences, presentations, meetings, lectures [2]. Figure 1 shows infrastructure of ad hoc networks.

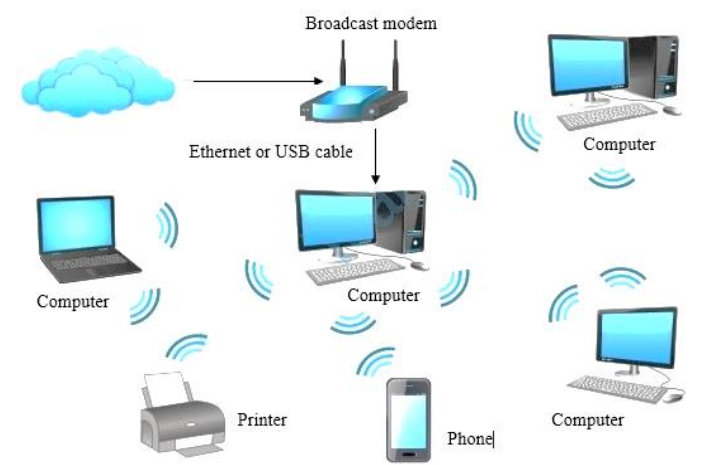

Fig 1. Infrastructure of Ad hoc networks

\section{II- ROUTING PROTOCOLS}

Routing can be defined as the process of sending data from source to destination. Routing is a key feature of MANETS because it enables messages to pass from node to another down to the target machine [3]. In MANETS, routing needs complex analysis to determine the optimal path. The routing protocol is basically designed to build routing 
tables to forward data to destination. Figure 2 depicts an example of multi-hop ad hoc network.

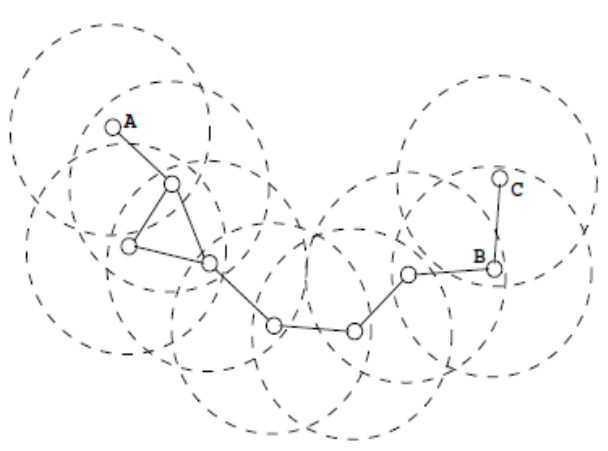

Fig 2. A multi-hop mechanism in ad hoc networks [4]

There are different routing protocols as a solution to the problem of routing in MANETs. Three commonly used classes to classify routing protocols in MANETs are [5]:

\section{1- Reactive routing protocols (on-demand) class}

Reactive protocols are such protocols that find a route only in case of data transmission. Reactive protocols are also called on-demand protocols because they seek routes only when it's required. Hence, they can significantly reduce the service traffic, because they seek routes only when it's required $[5,6]$.

\section{Pros and cons:}

- Network overhead increase just when querying for routes.

- Utilizing bandwidth just when required (when finding a path).

- Initial delay in traffic.

- Latency is increased.

Many on-demand routing protocols are conceptually simple and easily implemented, evaluated, and upgraded. Reactive routing can be characterized by the following protocols.

\section{Ad Hoc On-Demand Distance-Vector Routing protocol (AODV)}

AODV protocol reduced the number of official dispatches which constantly maintains a list of all routes. In this protocol, to discover the route to a destination node, the source node sends a request to its neighbors. In turn, neighbors transmit the request to their neighbors, until the package reaches the addressee or until the packet reaches an intermediate node having advanced routing destination information. Node discards those packets of route request that already were processed. This is done by using the route request packets sequence numbers. Using serial numbers also manages to avoid cycling routes. when an intermediate node passes the route request to their neighbors, they also record the route in its table about the node which came from the first node. This information recorded will exploit again to create a return route for the route reply packet (RREP). AODV requires using just symmetric communication channels $[7,8]$.

\section{Dynamic Source Routing protocol (DSR)}

This Protocol is a direct descendant of source routing scheme used in switched computer networks, for example, Token-Ring. DSR eliminates the periodic routing updates and has some advantages over the other protocols. Each packet carries a list of routers traversed on the way, so routing tables aren't required to be saved at each node as the route is clearly described in the package. DSR can support the bidirectional and unidirectional communication lines and establish routes on demand from the source node. The problem of dynamic source routing is broken into two separate tasks by the algorithm, the problem of route discovery and maintenance task.

Using DSR, the route request collects the list of addresses representing possible paths to a specific destination. Thus, when the demand reaches the destination, it can be returned to the source together with a recipient list therein that it can use in the reverse order [8].

\section{2- Proactive routing or table-driven routing protocols class}

Proactive protocols find the route before the need to transfer data arises. Proactive protocols are also called tabular ones. Proactive protocols make routing tables and periodically update them. In proactive routing protocols, each node keeps up at least one table contains the routing information to reach all other nodes within the network. Tabular protocols differ from each other by method of the topology change information distribution and the number of tables used for routing. The example of this class of protocols is Optimized Link-State Routing protocol (OLSR) [9].

Pros and cons:

- Network overhead is constant all the time.

- Information is forwarded in the whole network.

- Routes are always available.

- Latency is reduced.

\section{Optimized Link-State Routing protocol (OLSR)}

In OLSR protocol, there are three primary modules [9, 10]:

\section{A- Neighbor/link sensing}

In this module, it can detect neighbors and links by HELLO messages. The nodes send HELLO messages on a certain period. These 
messages are sent to determine the status of the links. Every message contains all the information about all neighbors. This makes a node within the network builds a routing table about its multiple hop neighbors.

\section{B- Optimized flooding/forwarding (Multi- Point Relaying- MPR)}

This module is the key idea behind the OLSR protocol to reduce the number of duplicate packet inside the network. Instead of all the nodes send the route packets to all neighbors, just a certain node, called Multipoint Relay (MPR) nodes, selected in the neighbor of source node, need to send route packets. Figure 3 depicts a comparison between a- Regular flooding and b- MPR flooding [10].

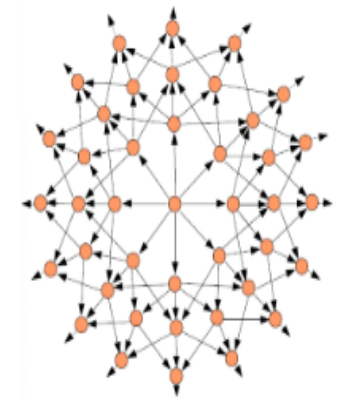

a- Regular flooding

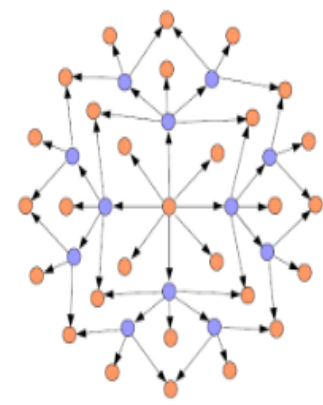

b- MPR flooding

Fig 3. Comparison between Regular and MPR flooding [10]

C-Link-State messaging and route calculation In this module, the nodes send link-state information to all nodes within the network. OLSR has two link-state optimizations:

- To reduce the set of nodes transmitting link-state messages, just MPRs nodes generate link-state messages

- To reduce the size of link-state messages, Just MPR selectors are declared in linkstate messages.

\section{3- Hybrid routing protocols class}

These protocols depending on the situation, use techniques such as reactive and proactive protocols choosing the most suitable of them. Group of protocols that use for routing a unification of nodes in the group are called hierarchical [5]. The examples of this class of protocols are Gathering-based routing protocol (GRP).

\section{Pros and cons:}

- Depends on amount/number of nodes activated.

- Suitable for large networks.

- Increase of Complexity.

- Latency is similar to reactive outside zone, and inside is low.
Gathering-based routing protocol (GRP):

In this protocol, the source node collects the information of network and then it can find routes to destination and directly transmit data. Thus, it can achieve fast transfer with less overhead. A destination query (DQ) packet is sent continuously to neighbors until reach to the destination, then the destination node will broadcast a packet called network information gathering (NIG) to its neighbors. Based on collected information, the source finds the best route and then instantly starts to transmit data [11].

\section{III- SIMULATION ENVIRONMENT}

The simulation was performed using OPNET modeler 14.5 simulation software in the identical environmental conditions. We check four protocols (AODV, DSR, OLSR and GRP) with 24 type of different scenarios based on the number and movement speed of the nodes in the network as shown in Table 1.

\section{Simulation Parameter}

Table 1- Simulation Parameter

\begin{tabular}{|c|c|}
\hline Parameter & Value \\
\hline Simulator & OPNET 14.5 \\
\hline No. of Scenarios & 24 \\
\hline Routing Protocol & OLSR, DSR, AODV, \\
\hline Network area & Campus (1000m*1000m) \\
\hline Channel & Wireless \\
\hline Traffic Type & TCP (FTP) \\
\hline Simulation time & $3600 \mathrm{sec}$ \\
\hline No. of nodes & $5,30.100$ \\
\hline Mobility of node & Random \\
\hline Speed & $10,30 \mathrm{~m} / \mathrm{s}$ \\
\hline
\end{tabular}

\section{Performance Metrics}

There are many of metrics to evaluate performance of the routing protocols. Every metric has a different effect of the overall network performance. In this paper, we evaluate four metrics (overhead, delay, network load, and throughput) to study their effect on the network performance. These metrics are important in evaluation of the routing protocols in a communication network. 
- Routing Overhead: Refers to a total amount of routing packets sent in the network.

- Network Throughput: Refers to a total amount of routing packets received in the network.

- End to End Delay: Refers to the time required to reach the packets from the source to the destination

- Routing load: Refers to the number of routing packets transmitted per data packet.

\section{Simulation Procedure}

The following steps provide a definition of setting up the topology used in our work:

- Create a Campus network of size $1 \mathrm{~km}$ x $1 \mathrm{~km}$

- From object palette, drag the wlan_server in the workspace.

- Drag the required number (according to the scenario) of wlan_ wkstn onto the workspace.

- Select (Auto-assign IPv4 addresses) for all subnet in the workspace.

- Choose the appropriate protocol for all subnet in the workspace.

- Drag the Application Config object from the object palette onto the workspace and then set it to model the high load FTP traffic.

- Drag the Profile Config object from the MANET object palette onto the workspace and then set the time from the start of the profile to start of the application.

- Drag the Mobility Config from the MANET object palette onto the workspace and then set the speed at which the mobile node will be moving.

- Collect Statistics.

\section{IV- SIMULATION RESULTS AND}

ANALYSIS

This section depicts the results obtained during the simulation. Results were collected for different protocols in terms of all performance metrics previously mentioned. At last we compare the results of routing protocol.

In Figure 4, we observe that the mobility does not effect on the amount of routing traffic with the network comprising 5 and 30 nodes in all protocols. But with 100 nodes, we observe that there is small effect when changing the speed from 10 to $30 \mathrm{~m} / \mathrm{s}$ in AODV and DSR protocols.
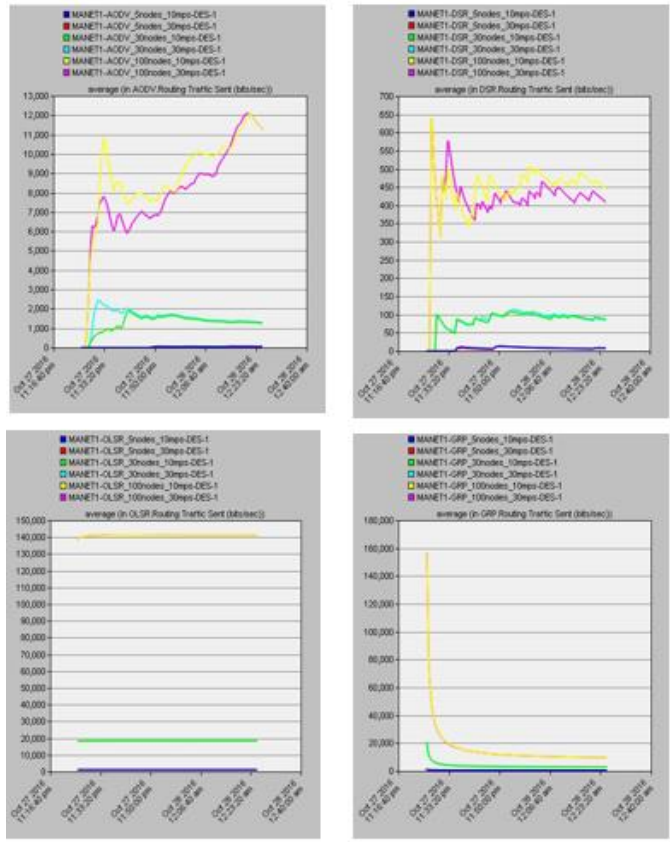

Fig 4. Routing overhead in individual protocols (OLSR, DSR, AODV, and GRP)

In Figure 5, we observe that DSR and AODV protocols have the best performance in terms of routing overhead because they send the least amount of routing traffic whilst OLSR protocol have a worst performance with the highest amount of routing traffic sent.
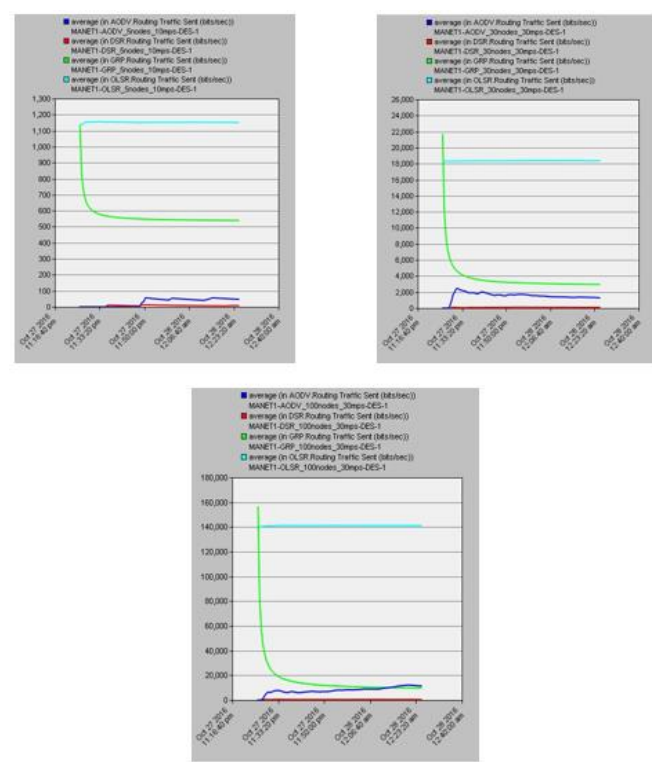

Fig 5. Routing overhead in protocols (OLSR, DSR, AODV, and GRP)

From the Figures 6 by looking at the throughput performance between protocols, it is clear that throughput in OLSR is highest in all scenarios. At the same time, we observe that in the small network, DSR outperformed 
AODV whilst AODV outperformed DSR in the larger network when the nodes were moving at $30 \mathrm{~m} / \mathrm{s}$.
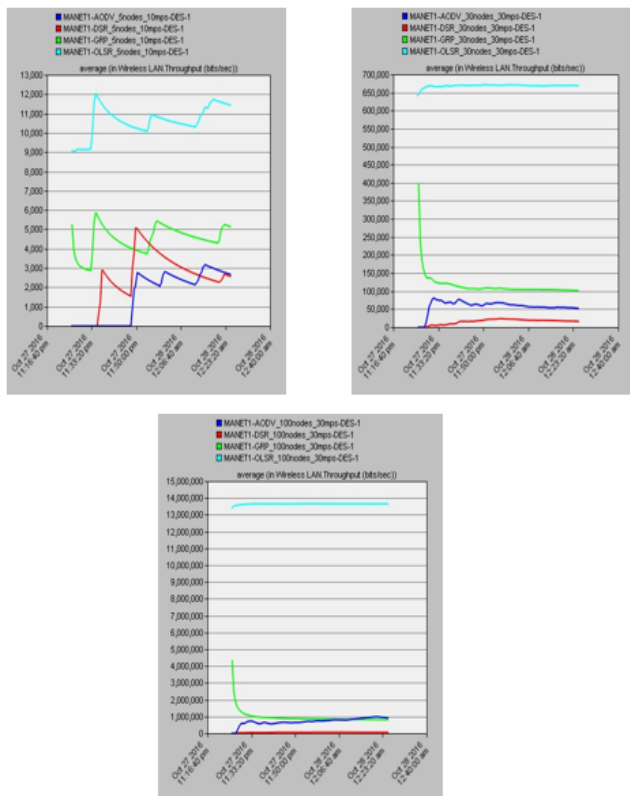

Fig 6. Throughput in protocols (OLSR, DSR, AODV, and GRP)

In Figure 7, we observe that OLSR has the lowest End to End packet delay in all scenarios due to its proactive characteristics. As for other protocols, we also observe that GRP outperformed AODV and DSR in the smaller network whilst AODV compete with GRP and OLSR in large networks with high speed.
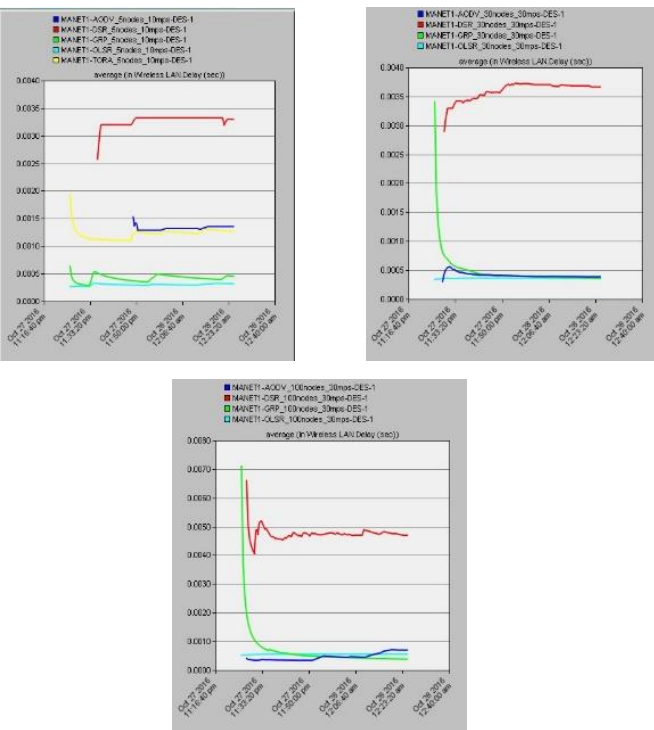

Fig 7. End to End Delay in protocols (OLSR, DSR, AODV, and GRP)

In Figure 8, we observe that, in small networks, all Protocols compete with each other in terms of routing load. While in large networks, OLSR has highest routing load compared with other protocols.

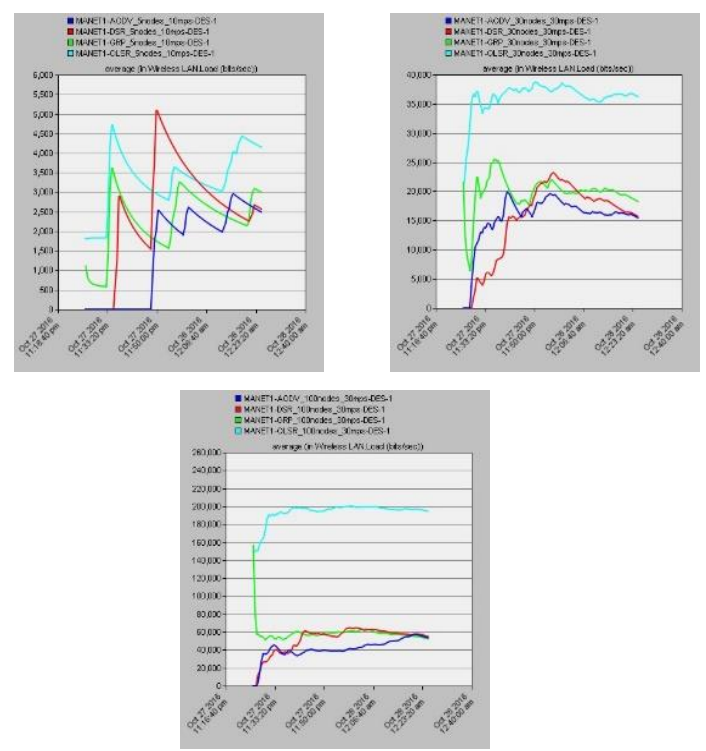

Fig 8. Routing load in protocols (OLSR, DSR, AODV, and GRP)

\section{Discuss and compare the results}

As seen above in our simulation results, each protocol, in comparison with others, has its advantages and disadvantages that make them suitable for specific scenarios.

- In proactive routing protocol (OLSR,) routing information is always available with minimal delay, but at the same time, all nodes within the network need to update this information causing extra control overhead. Thus, these protocols consume high power and high bandwidth to keep routing information up-to-date.

- Reactive protocols (AODV and DSR) are contrast to previous protocols where the nodes seek routes only when required with long delay and less overhead. Thus, these protocols consume low power and low bandwidth compared to proactive protocols.

- In hybrid protocols (GRP), routing more scalable and efficient where they combine attributes of reactive and proactive protocols. Delay is lower for intra-zone and higher for inter-zone. Overhead is less than in proactive protocols. Therefore, these protocols consume less power and less bandwidth compared to proactive protocols but high power and high bandwidth compared to reactive protocols. Table 1 provides a comparison between ad-hoc routing protocols. 
Table 1 Comparison between Proactive, Reactive and Hybrid protocols.

\begin{tabular}{|c|c|c|c|c|c|c|}
\hline Type & Delay & Latency & Orerhead & Bandridth & $\begin{array}{c}\text { Porrer } \\
\text { consumption }\end{array}$ & Scalability \\
\hline $\begin{array}{l}\text { Proactire } \\
\text { (OLSR) }\end{array}$ & Lorver & Low & High & High & High & Low \\
\hline $\begin{array}{c}\text { Reactire } \\
\text { (DSR,ADDV) }\end{array}$ & Higher & High & Lorr & Low & Low & $\begin{array}{l}\text { Not sutuble } \\
\text { rith large } \\
\text { Networks }\end{array}$ \\
\hline $\begin{array}{l}\text { Hybrid } \\
\text { (GRP) }\end{array}$ & Medium & Medium & Medium & Medium & Medium & $\begin{array}{c}\text { Designed for } \\
\text { large } \\
\text { networks }\end{array}$ \\
\hline
\end{tabular}

\section{V- CONCLUSIONS}

In this research, the performance of three classes of MANET routing protocols (Reactive, Proactive and Hybrid) have been evaluated to ensure better evaluation unlike many of previous studies that evaluated the performance of one or two classes of protocols and choice the best of them such as $[2][5][6][7][8][11]$.

We selected four protocols AODV, DSR, OLSR, and GRP for evaluate them in terms of four metrics (routing overhead, network throughput, packet end to end delay and routing load) using the simulation environment OPNET 14.5.

The study of these routing protocols shows that the mobility has no clear effect on the network performance of the routing protocols according to our simulation results.

OLSR has best performance than of all four protocols in terms of throughput and packet end-to-end delay but it has the worst performance in the routing overhead. Therefore, OLSR routing protocol is suited for small networks.

DSR is the opposite of OLSR whereas it outperformed in cases where OLSR does not perform well.

AODV is better than all other protocols used in our simulation in terms of load networks.

GRP, OLSR and AODV are almost competing in terms of end to end delay in large networks with high speed but in small networks GRP, OLSR is better than others.

The basic differences between ad hoc routing protocols (reactive, proactive and hybrid) in how to discover the routes and maintenance them.

Finally, we conclude that there is no single protocol is superior in all respects and cannot naming any of the protocols is best than others. The performance of routing protocols differs with the network and choice of suitable routing protocol according to the network. Therefore, the planned use of the network is that determines the selection of accurate protocol.

\section{REFERENCES}

[1] Soni, S., \& Srivastava, S. (2016). Survey of Quality of Service Routing Protocol in MANET. International Journal of Computer Applications, 136(13), 6-10. doi:10.5120/ijca2016908541.

[2] Horane, A., Jeyakumar, A. \& Patkar, S. (2013). Different routing protocol for MANET: A survey. International Journal of Engineering Research and Applications (IJERA), 3(2), 1191-1195.

[3] Kondaiah, B. (2014) A Comparative Analysis of Pro-active Routing Protocols in MANET. IJCSMC, 3(6), $144-151$.

[4] Piconet II - A Wireless Ad Hoc Network for Mobile Handheld Devices. (n.d.). Retrieved October 10, 2016, from http://piconet.sourceforge.net/thesis/main.h $\underline{\mathrm{tml}}$.

[5] $\overline{\text { Rai }}$ H. (2014), A Comparison of Performance Metrics for Various Routing Protocols in MANET. IJCSMC, 3(6), 239 $-246$.

[6] Channappagoudar, M. B., \& Venkataram, P. (2016). Performance evaluation of mobile agent based resource management protocol for MANETs. Ad Hoc Networks, 36, 308-320. doi:10.1016/j.adhoc.2015.08.008.

[7] Heena and Goyal D. ,2014, "Congestion control using enhance AODV (EAODV) routing mechanism in MANET", IJCSMC, 3(8), $555-565$.

[8] Munisha, Goyal D. (2014). Simulation and performance analysis of AODV protocol in MANET. IJCSMC, 3(7), $474-486$.

[9] Sumari.P. \& Al-Omari. S.A. (2010). An overview of mobile Ad-Hoc Networks for the existing protocols and applications. International journal on application of graph theory in wireless ad hoc networks and sensor networks (Graph-Hoc). 2(1), 87110.

[10] Introduction to wireless networking. (n.d.). Retrieved October 23, 2016, from http://perso.crans.org/raffo/papers/phdthesi s/thesisch1.html.

[11] Chavhan A. D. \& Asole. S.S. (2014). Comparative Analysis and Performance Evaluation of MANET Routing Protocols", IJCSMC, 3(12), 369 - 375. 